\title{
UV-Vis Spectrophotometry and Chemometrics as Tools for Recognition of the Biochemical Profiles of Organic Banana Peels (Musa sp.) According to the Seasonality in Southern Brazil
}

\author{
Susane Lopes ${ }^{1(\sqrt{(})}$, Rodolfo Moresco ${ }^{1}$, Luiz Augusto Martins Peruch ${ }^{2}$, Miguel Rocha $^{3}$, \\ and Marcelo Maraschin ${ }^{1}$ \\ ${ }^{1}$ Plant Morphogenesis and Biochemistry Laboratory, Federal University of Santa Catarina, \\ Florianopolis, Brazil \\ susane. lopes@ufsc.br \\ 2 Agricultural Research and Rural Extension Company of Santa Catarina, Criciúma, Brazil \\ ${ }^{3}$ School of Engineering, Centre Biological Engineering, University of Minho, Braga, Portugal
}

\begin{abstract}
Banana (Musa sp.) has received wide interest in popular and scientific medicine because of its rich composition in bioactive metabolites, e.g., phenolic compounds, found in interesting concentrations in its peel. Banana peel is a residue that is under-exploited by the industry. Thus, with the intention to give a destination to this by-product towards health care or cosmetics industries, we evaluated its aqueous extract (AE) as a source of bioactive phenolic compounds, aiming at to apply them in future studies of biological activities. For that, in this study samples of banana peels were chemically profiled throughout the year to identify the best harvest time of those biomasses regarding their phenolic composition. In this sense, we used additional information on the chemical heterogeneity of the samples determined by the seasoning, through a set of analytical and climatic data to elaborate chemometric models, supported by bioinformatics tools. Through PCA and HCA analyzes, it was detected that low temperatures; normally observed in winter; strongly modulate the banana metabolism, leading to increased amounts of phenolic compounds, and improving the antioxidant activity of the banana peel AE. The samples collected during the months of winter showed a similar profile and a relatively high concentration of phenolic compounds with potential for future studies of biological activities.
\end{abstract}

Keywords: Banana - Musa sp. · Peels · Phenolic compounds · Antioxidant activity $\cdot$ Spectrophotometry $\cdot$ Chemometrics $\cdot$ Seasonality $\cdot$ Metabolic profile

\section{Introduction}

Banana (Musa sp.) is an edible fruit grown in tropical and subtropical regions with seasonal chemical variation in its pulp and peel composition due to the effect of climatic factors, e.g., rainfall and temperature [1]. In Brazil, one of the largest banana producers worldwide [2], the peel is the main by-product of the banana industrial processing, 
accounting for approximately $38 \%$ of the total weight of the fruit. This residual biomass is considered a waste with low economical value [3]. Studies have shown that banana is a good source of carbohydrates, mostly starch, minerals, vitamin B6, natural antioxidants $[4,5]$, as well as carotenoids and biogenic amines [6].

In the last few decades, banana has been evaluated by scientific and medicinal interests as an important source of bioactive compounds, such as flavonoids, anthocyanins, condensed tannins, and biogenic amines. These compounds have been extensively documented for their actions in promoting health in the reduction of chronic diseases, e.g., cancer, cardiovascular dysfunction, and muscular degeneration [4], besides the antibacterial, antiulcerogenic, antihypertensive antidiabetic, and antioxidant activities [7].

Phenolic compounds are secondary metabolites responsible for several of these therapeutic properties, mainly due to their antioxidant potential, and obtained in interesting concentrations in banana peels [3]. In local and traditional Brazilian medicine, the banana peel has a useful history to promote the healing of wounds mainly by burns when used topically [6], assigning an interesting destination to this residual biomass. Thus, our group aims to recover the phenolic compounds from the banana peel and confer a use to this residue. In this study, we determined the chemical profiles of AEs of banana peels collected over the year to better understand their seasonal heterogeneity. For that, we used a set of analytical and climatic data to build chemometric models, supported by bioinformatics tools. By applying multivariate statistical techniques (principal component analysis - PCA and hierarchical clustering analysis - HCA), we investigated the influence of climatic variables and their relation with biosynthesis of phenolic compounds and their antioxidant activity in samples of banana peel, collected over the seasons in the Santa Catarina State, southern Brazil. This strategy aims to obtain additional information about the biochemical heterogeneity of the samples caused by the seasonality, in order to select the best samples for future studies of biological activities, also driving eventual technological usage.

\section{Materials and Methods}

\subsection{Banana Samples and Processing of Plant Material}

Samples of banana peels (Musa sp., cv. Prata Anã) were monthly collected (February 2015 to January 2016) from an orchard agroecologically managed, and provided by the Agricultural Research and Rural Extension Company of Santa Catarina (EPAGRI -

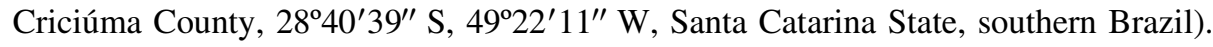
The banana samples were sanitized in running water and dried with a paper towel. Then, the fruit peels were manually removed and dried in an oven $\left(45^{\circ} \mathrm{C}\right)$, with air flow, until constant weight. The dry biomass was packed in polyethylene bags and stored at $-20^{\circ} \mathrm{C}$. Dried banana peels samples $(0.5 \mathrm{~g})$ were added of $7.5 \mathrm{ml}$ distilled water and incubated (water bath, $37^{\circ} \mathrm{C}, 30 \mathrm{~min}$ ), followed by centrifuging and recovering of the supernatant as the aqueous extract (AE) according to Pereira (2014) [8]. 


\subsection{Spectrophotometric Analysis}

The AE's chemical profiles were determined through UV-Vis scanning spectrophotometry (Gold Spectrum lab 53 UV-Vis spectrophotometer, BEL photonics, Brazil) over a spectral window of 200 to $800 \mathrm{~nm}$ ( $1 \mathrm{~nm}$ resolution/data point). The content of total phenolic compounds was determined according to Randhir, Shetty and Shetty (2002) [9], while the total flavonoids amounts followed the methodology proposed by Woisky and Salatino (1998) [10]. The ability of the AE's to scavenge the 1, 1-diphenyl-2picrylhydrazyl (DPPH) free radical was determined based on the method of Ribeiro et al. (2008) [11].

\subsection{Statistical and Chemometric Analysis}

Data were collected, summarized, and submitted to analysis of variance (ANOVA) followed by the post-hoc Tukey's test $(p<0.05)$ for mean comparison. All procedures were performed in triplicate, in three independent experiments $(n=9)$. The processing of the spectrophotometric profile considered the definition of the spectral window of interest (200-800 nm), baseline correction, normalization, and optimization of the signal/noise ratio (smoothing). The processed data set was subjected to multivariate statistical analysis, by applying principal component analysis (PCA) and clustering methods, as well as predictive machine learning tools. All analyses were supported by scripts written in the $\mathrm{R}$ language using tools developed by our research group (the specmine package) [12] and some functions from the packages Chemospec [13], HyperSpec [14], and ggplot2. All R scripts, raw data, and additional chemometric analysis are available in supplementary material, in http://darwin.di.uminho.pt/pacbb2017/bananapeels, as well as the data analysis report automatically generated from the $\mathrm{R}$ scripts using the features provided by R Markdown. This allows anyone to fully reproduce and document the experiments.

\section{Results and Discussion}

Phenolic compounds are secondary metabolites found in plants with important biological activities. We propose to recover these compounds by producing AE's of banana peels collected over the seasons, to select the best sample for future biological assays. Initially, biochemical (total contents of phenolic compounds and flavonoids, and the inhibition activity of the DPPH radical) and spectrophotometric (absorbance at $\lambda=200$ $800 \mathrm{~nm}$ ) assays were done, followed by chemometric analysis.

Initial exploratory analysis, with simple descriptive statistics and boxplots of the main variables, indicated differences, namely in the total phenolics and flavonoids concentrations, which appear to be higher in the winter samples. By performing a linear regression relating phenolic and flavonoid contents and climatic factors, the results indicated that lower temperatures increased the amounts of total phenolics and flavonoids in banana peels. This trend was clearer for the total phenolics ( $\mathrm{mg}$ gallic acid equivalent/g dry peels; i.e., $\mathrm{mg} \mathrm{GAEq} / \mathrm{g})$, where for the minimum temperature $\left({ }^{\circ} \mathrm{C}\right)(\mathrm{Fig} .1)$ the variance explained $\left(R^{2}=0,702\right)$ is over $70 \%$, with a quite low p-value $\left(4 \times 10^{-4}\right)$. 


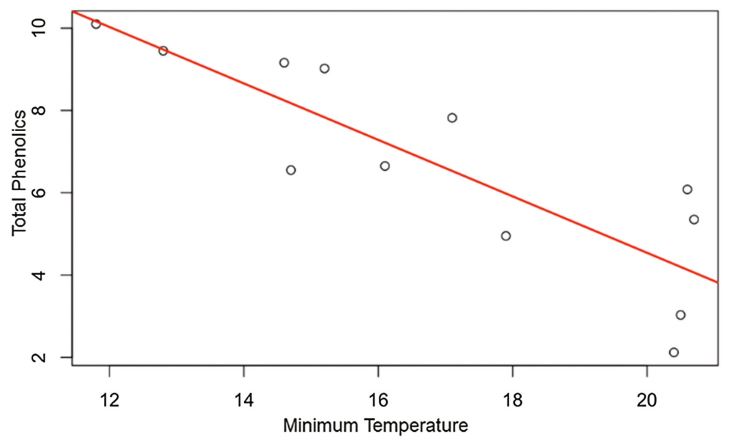

Fig. 1. Linear regression overlapping the data points (sample/month) of the variables total phenolic compounds (mg GAEq/g) vs. minimum temperature $\left({ }^{\circ} \mathrm{C}\right) . \mathrm{R}^{2}(0,702)$ is above $70 \%$.

Further, the Pearson correlations for the dataset were calculated, corroborating the previous findings, where a significant relationship $(\mathrm{r}=-0.854)$ between the minimum temperature and the total phenolic compounds was detected (Fig. 2). In a similar statistical approach, the variables air moisture, total rainfall, and insolation (amount of solar energy $/ \mathrm{cm}^{2} / \mathrm{min}$ ) did not show significant relationship with the biochemical variables.

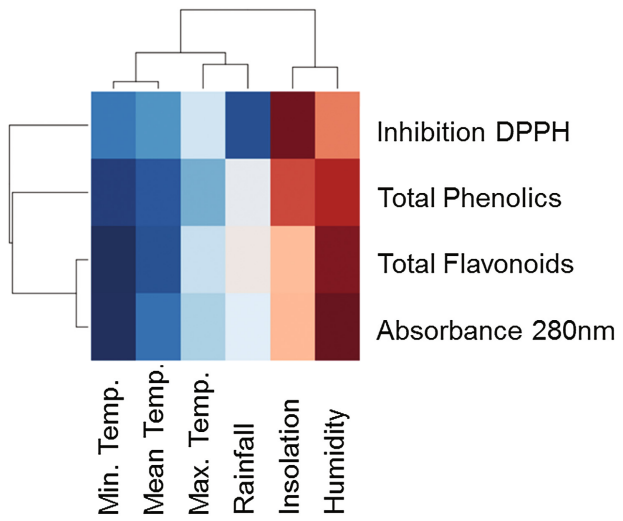

Fig. 2. Pearson correlation between biochemical and climatic variables. The correlation increases in blue and decreases in red.

In a second stage, the UV-Vis dataset $(\lambda=200-800 \mathrm{~nm})$ of the AE's was preprocessed, where an offset correction and smoothing were applied. All the spectral profiles $(\lambda=200-800 \mathrm{~nm})$ of AE's showed absorbances unity (Au) in the spectral window typical of phenolics $(\lambda=280-320 \mathrm{~nm})$, indicating that the extraction system was able to recover the secondary metabolites from the residual biomass. In addition, the spectral profiles were similar, suggesting a homogeneous chemical composition among samples over the seasons (Fig. 3). 


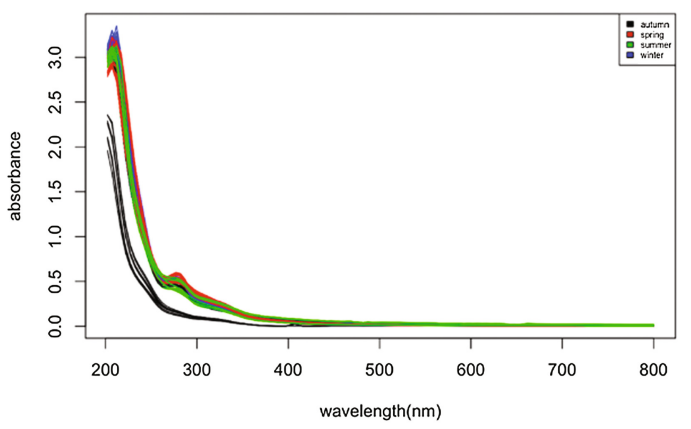

Fig. 3. UV-Vis spectroscopic profiles $(\lambda=200-800 \mathrm{~nm}-\mathrm{Au})$ of 12 representative samples of aqueous extracts of banana peels, collected during the seasons of 2015 (summer, fall, winter and spring) and 2016 (summer) in southern Brazil.

Further, PCA of the spectroscopic profiles, an unsupervised multivariate statistical technique, revealed a clear seasoning effect on the grouping of samples. PC1 $(58.1 \%)$ and PC2 (37.3\%) comprised $95.4 \%$ of the total variance of the dataset, making possible to explain the data variability with a few latent orthogonal variables. Overall, the results showed a certain degree of discrimination among the samples over the seasons regarding their chemical composition (Fig. 4).

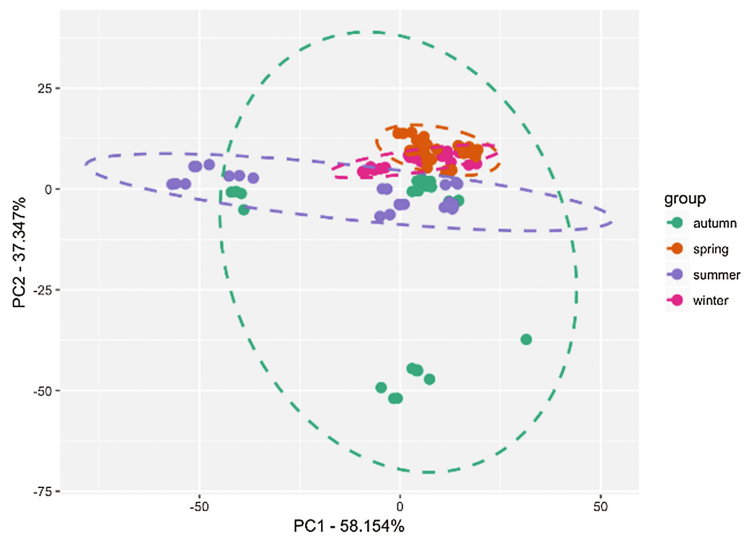

Fig. 4. Score scatter plot of the UV-Vis spectral data $(\lambda=200-800 \mathrm{~nm})$ on the PC1 and PC2 axes of samples $(n=108)$ of aqueous extracts of banana peels. Each set of points of the same coloration represents a season.

In a follow-up experiment, hierarchical cluster analysis (HCA) was applied to the spectroscopic dataset and a better sample discrimination seems to be found in comparison to PCA. Again, the findings revealed that unsupervised methods end up merging the data into a pattern that fits well into the natural groups in the sample season (Fig. 5). 


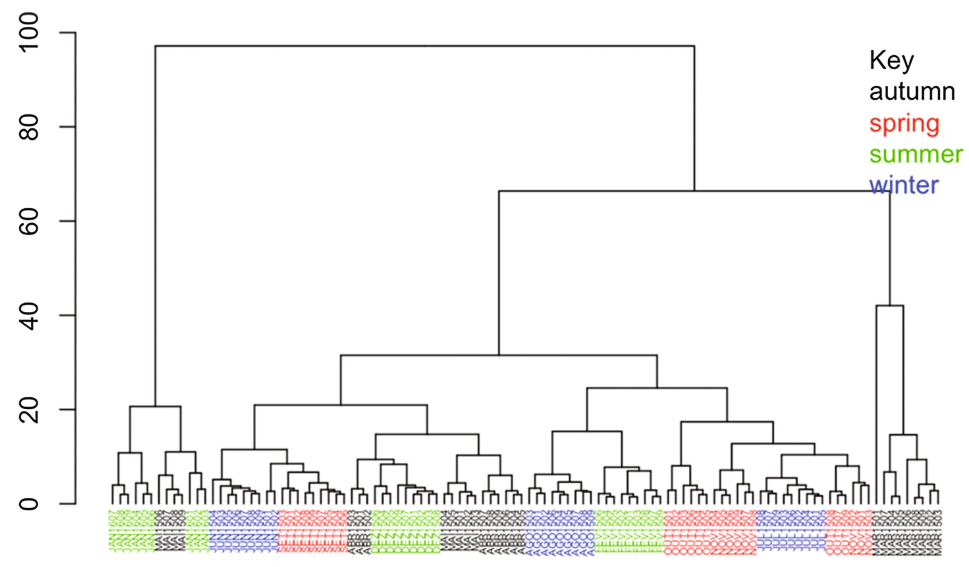

Fig. 5. Hierarchical cluster analysis (HCA) of the UV-Vis absorbances $(\lambda=200-800 \mathrm{~nm})$ of aqueous extracts of banana peels.

Since phenolic compounds are usually the majoritarian ones in AE's of banana peels, we further performed PCA and HCA aiming at to extract additional information correlating the seasonality with the maximum absorption of peaks of those secondary metabolites $(\lambda=280-320 \mathrm{~nm})$. In the PCA, PC1 (96.9\%) and PC2 (2.8\%) explained $99.7 \%$ of the total variance of the data set, but an improved sample discrimination regarding the full spectroscopic dataset $(\lambda=200-800 \mathrm{~nm})$ was not achieved.

Thus, HCA using the same spectral dataset was done affording a better discrimination (Fig. 6). From the root, the first split separates the MAR/2015 samples (fall season) from the remaining, which corroborates the biochemical assays where the lowest amounts of secondary metabolites were found. Navigating the tree downwards, the samples from the second group are clustered by similarity in 3 groups, which have a majority of winter, summer and spring samples in each. Winter samples are grouped integrally, giving a similarity between the JUN/15, JUL/15 and AUG/15

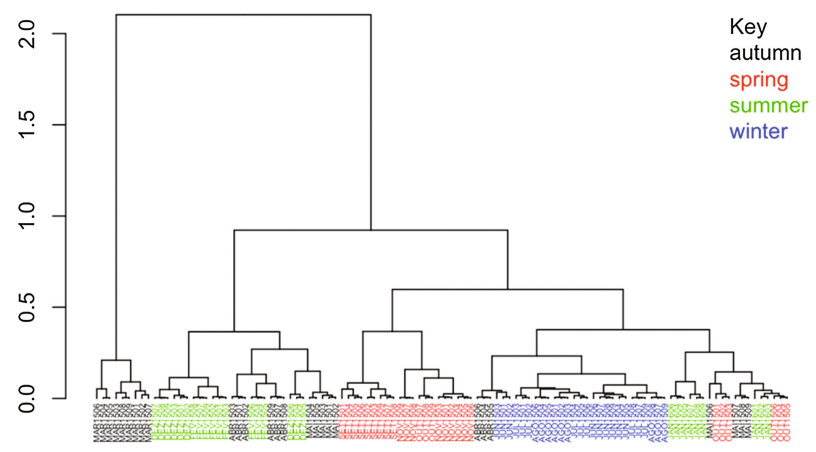

Fig. 6. Hierarchical cluster analysis of the UV-Vis absorbance region of phenolic compounds $(\lambda=280-320 \mathrm{~nm})$ of crude aqueous extract of banana peels. 
samples with respect to the spectral region of interest. In the right, we can see a cluster that mixes summer, autumn, and spring samples.

\section{Conclusions}

The analytical approach employed in this work, supported by bioinformatics tools, allowed a better understanding of the chemical variability of the banana peels collected during seasons associated to the climatic factors. Low temperatures typically found in the winter were determinant to modulate the banana metabolism for the production of increased amounts of phenolic compounds, also improving the antioxidant activity of AE's. Thus, PCA and HCA demonstrated a discrimination of samples collected in the winter as promising for future biological studies. Besides, HCA allowed identifying autumn-collected samples, i.e., MAR/2015, which showed reduced amounts of bioactive secondary metabolites.

Acknowledgements. To CNPq (National Counsel of Technological and Scientific Development) for financial support (Process n ${ }^{\circ} 407323 / 2013-9$ ), to CAPES (Coordination for the Improvement of Higher Education Personnel), and EPAGRI (Agricultural Research and Rural Extension Company of Santa Catarina). The research fellowship from CNPq on behalf of M. Maraschin is acknowledged. The work is partially funded by Project PropMine, funded by the agreement between Portuguese FCT and Brazilian CNPq.

\section{References}

1. Anyasi, T.A., Jideani, A.I.O., Mchau, G.A.: Morphological, physicochemical, and antioxidant profile of noncommercial banana cultivars. Food Sci. Nutr. 3, 221-232 (2015)

2. EMBRAPA (Empresa Brasileira de Pesquisa Agropecuária), Brasília, Brazil. https:// www.embrapa.br/mandioca-e-fruticultura/cultivos/banana. Accessed 08 Feb 2017

3. Vu, H.T., Scarlet, C.J., Vuong, Q.V.: Optimization of ultrasound-assisted extraction conditions for recovery of phenolic compounds and antioxidant capacity from banana (Musa cavendish) peel. J. Food Process. Preserv. 40, 1-14 (2016)

4. Yuan, Y., Zhao, Y., Yang, J., Jiang, Y., Lu, F., Jia, Y., Bao, Y.: Metabolomic analyses of banana during postharvest senescence by ${ }^{1} \mathrm{H}$-high resolution-NMR. Food Chem. 218, 406412 (2017)

5. Tsamo, C.V.P., Andre, C.M., Ritter, C., Tomekpe, K., Newilah, G.N., Rogez, H., Larondelle, Y.: Characterization of Musa sp. fruits and plantain banana ripening stages according to their physicochemical attributes. J. Agric. Food Chem. 62, 8705-8715 (2014)

6. Pereira, A., Maraschin, M.: Banana (Musa sp.) from peel to pulp: Ethnopharmacology, source of bioactive compounds and its relevance for human health. J. Ethnopharmacol. 160, 149$163(2015)$

7. Tsamo, C.V.P., Herent, M., Tomekpe, K., Emaga, T.H., Quetin-Leclercq, J., Rogez, H., Larondelle, Y., Andre, C.: Phenolic profiling in the pulp and peel of nine plantain cultivars (Musa sp.). Food Chem. 167, 197-204 (2015) 
8. Pereira, A.: Determinação do perfil químico e da atividade cicatrizante de extratos de casca de banana cultivar prata anã (Musa sp.) e o desenvolvimento de um curativo para pequenas lesões. 223 f. Tese (Doutorado em Biotecnologia e Biociências) - Universidade Federal de Santa Catarina, Florianópolis, Santa Catarina (2014)

9. Randhir, R., Shetty, P., Shetty, K.: L-DOPA and total phenolic stimulation in dark germinated fava bean in response to peptide and phytochemical elicitors. Process Biochem. 37, $1247-$ $1256(2002)$

10. Woisky, R.G., Salatino, A.: Analysis of propolis: some parameters and procedures for chemical quality control. J. Apic. Res. 37, 99-105 (1998)

11. Ribeiro, S.M.R., Barbosa, L.C.A., Queiroz, J.H., Knodler, M., Schieber, A.: Phenolic compounds and antioxidant capacity of Brazilian mango (Mangifera indica L.) varieties. Food Chem. 110, 620-626 (2008)

12. Costa, C., Maraschin, M., Rocha, M.: An R package for the integrated analysis of metabolomics and spectral data. Comput. Methods Programs Biomed. 129, 117-124 (2015)

13. Hanson, A.B.: ChemoSpec: an R package for chemometric analysis of spectroscopic data and chromatograms (Package Version 1.51-0) (2012)

14. Beleites, C.: Import and export of spectra files. Vignette for the R package hyperSpec (2011) 